\title{
WAVE FUNCTIONS OF DISORDERED TWO-DIMENSIONAL ELECTRON GAS IN STRONG MAGNETIC FIELD*
}

\author{
K.I. WYSOKIŃSKI \\ Institute of Physics, M. Curie-Skłodowska University \\ Radziszewskiego 10a, 20-031 Lublin, Poland \\ AND W. BRENIG \\ Physics Department - T30, Technische Universität München \\ W-8046 Garching, Germany
}

\begin{abstract}
The wave functions of the disordered two-dimensional electron gas (2DEG) placed in strong perpendicular magnetic field are calculated numerically. Even though the purpose of this work is mainly pedagogical, we shall shortly discuss our motivation which is possible application of the results to the calculations of the conductivity tensor in transition region between consecutive plateaus of the Hall conductivity in the quantum Hall effect.
\end{abstract}

PACS numbers: $72.10 . \mathrm{Bg}, 73.20 . \mathrm{Dx}$

\section{Introduction and motivation}

The phenomenon of resistance quantisation [1] relies on the existence of both extended i.e. current carrying and localised (or bound) states [2]. It is the compensation theorem [3] which ensures the current conservation and resistance $\rho_{x y}=h /\left(n e^{2}\right), n=1,2 \ldots$ quantization.

As a result the appearance of plateaus, their height and precision of quantization is believed to be correctly understood. The same can not be said about the transition region between plateaus where $\rho_{x y}$ viewed as a function of magnetic field is not quantised and where $\rho_{x x}$ takes on nonzero values implying that dissipation appears in the system.

*This work is supported in part by Ministry of National Education under the contract II 1.9.P/04/063.9. 
Recently we have derived a formula by which the calculation of the conductivity $\sigma_{x x}$ in conditions of quantum Hall effect has been reduced to the calculation of the scattering matrix [4]. This result can easily be obtained from the equation of motion for the momentum operator $\Pi=p-q_{\mathrm{e}} \boldsymbol{A}$ of an electron $\left(q_{\mathrm{e}}=-e\right)$ in magnetic field described by the vector potential $\boldsymbol{A}$. From the equality $\langle\alpha|[H, \boldsymbol{\Pi}]| \alpha\rangle=0$ valid for states $|\alpha\rangle$ being eigenstates of $H$, we get (for notation see [4]) the expressions for the average velocities $u^{y}(n k)=\left\langle n k+\left|v^{y}\right| n k+\right\rangle, u^{x}(n k)=\left\langle n k+\left|v^{x}\right| n k+\right\rangle$, ( $v=p / m, n$ denotes Landau band, $k$ is wave vector)

$$
\begin{aligned}
& u^{y}(n k)=-\frac{E}{B}+\frac{2}{\hbar q_{\mathrm{e}} B} \operatorname{Im} \sum_{m p}\langle n k+|V| m p\rangle\left\langle m p\left|p_{x}\right| n k+\right\rangle, \\
& u^{x}(n k)=-\frac{2}{\hbar q_{\mathrm{e}} B} \operatorname{Im} \sum_{m p}\langle n k+|V| m p\rangle\left\langle m p\left|p_{y}\right| n k+\right\rangle .
\end{aligned}
$$

The last formula can further be simplified and the final result reads

$$
u^{x}(n k)=-\frac{2}{q_{\mathrm{e}} B} \sum_{m p}(k-p)|\langle n k+|V| m p\rangle|^{2} \delta\left(\varepsilon_{m p}-\varepsilon_{n k}\right) .
$$

To calculate velocities and also conductivities from the above formulae one needs the functions $|n k+\rangle$ by definition being eigenfunctions of $H$. These are scattering, extended or current carrying states we are going to calculate in this work. In impure 2DEG in magnetic and electric fields both extended and localised states are expected to exist. In the scattering approach there is no problem in distinguishing between extended and localised state - question not resolved in a satisfactory way theoretically for a 2-dimensional impure system in strong $B$ field. Another point which motivates our study refers to finite temperature behaviour of $\sigma_{x x}$. As it is well-known the states in a Landau band of a clean system in $B$ and $\boldsymbol{E}$ fields are plane wave like in current direction $(y)$ and gaussian localised in electric field direction $(x)$. In a disordered system (or part of it) they change their characteristics and become, as we will see later "partially" extended in $x$ direction. Assuming the states in transition region to be extended in $y$ and power low localised, $\phi \propto x^{-S}$ in $x$ direction one finds hopping contribution to $\sigma_{x x} \propto T^{(2 S-3)}(2 S>3)$. It would be desirable to have experimental data for $T$-dependence of $\sigma_{x x}$ to check these predictions.

Other work known from literature dealing with structure of states in conditions of QHE concentrates on the exact diagonalization of the Hamiltonian, or makes quasiclassical approximations [5]. 


\section{The structure of states}

Let the Hamiltonian of the system be given by [4]:

$$
H=\Pi^{2} / 2 m-q_{\mathrm{e}} E x+V(r)=H_{0}+V(r),
$$

where $\Pi=p-q_{\mathrm{e}} \boldsymbol{A}, \boldsymbol{A}=B(0, x, 0)$, and eigenfunctions of $H_{0}$ be $\varphi_{n k}(r)$. The current carrying states (scattering states) $\psi_{\varepsilon}(r)$ at energy $\varepsilon$ are the solutions of the Lippmann-Schwinger equation

$$
\psi_{\varepsilon}(r)=\varphi_{\varepsilon}(r)+\int \mathrm{d} r^{\prime} G_{\varepsilon}\left(r, r^{\prime}\right) V\left(r^{\prime}\right) \psi_{\varepsilon}\left(r^{\prime}\right)
$$

where $G_{\varepsilon}\left(r, r^{\prime}\right)$ is the unperturbed Green's function in $r$ representation. For the Hamiltonian $H_{0}$ given by (3) and for $\varepsilon$ belonging to the lowest Landau band it can be expressed in closed form in terms of complex error function $w(z)$ :

$$
G_{\varepsilon}^{0}\left(r, r^{\prime}\right)=\frac{-\mathrm{i} \sqrt{\pi}}{e E l} P_{0}\left(r, r^{\prime}\right) w\left(z_{\varepsilon}\right),
$$

where

$$
P\left(r, r^{\prime}\right)=\frac{1}{2 \pi l^{2}} \exp \left[-\left(\frac{r-r^{\prime}}{2 l}\right)^{2}-\mathrm{i} \frac{y-y^{\prime}}{l}\left(\frac{x+x^{\prime}}{l}-\frac{e E l}{\hbar \omega_{\mathrm{c}}}\right)\right]
$$

and with $\varepsilon=\varepsilon_{0}(q)$

$$
z_{\varepsilon}=z_{q}=\frac{x+x^{\prime}}{2 l}-\mathrm{i} \frac{y-y^{\prime}}{2 l}+q l+\frac{e E l^{2}}{\hbar \omega_{\mathrm{c}}} .
$$

The bound states exist at energies $\varepsilon$ outside the allowed parts of $H_{0}$ spectrum. Here the nonhomogeneity term $\varphi_{n k}(r)$ in (4) vanishes and

$$
\psi_{\varepsilon}(r)=\int G_{\varepsilon}\left(r, r^{\prime}\right) V\left(r^{\prime}\right) \psi_{\varepsilon}\left(r^{\prime}\right) \mathrm{d} \boldsymbol{r}^{\prime}
$$

This constitutes condition for the energies $\varepsilon$ of bound states. What we need is the expression for the Green's function valid for $\varepsilon$ in gaps of the spectrum. Assuming the Landau bands to be much narrower than $\hbar \omega_{0}$ (valid for weak electric fields and not too wide - in $x$ direction - samples) we get

$$
G_{\varepsilon}^{b}\left(r, r^{\prime}\right)=\frac{1}{2 \pi l^{2}} P_{0}\left(r, r^{\prime}\right) \sum_{n=0}^{\infty} \frac{L_{n}\left(\frac{\left(r-r^{\prime}\right)^{2}}{2 l^{2}}\right)}{E-\hbar \omega_{\mathrm{c}}(n+1 / 2)}
$$

which is related to confluent hypergeometric function ${ }_{1} F_{1}(a, b ; x)$.

\section{Results}

We have solved equations (4) and (5) numerically for the short ranged random impurity potential given by

$$
V(r)=\sum_{i}^{N} V_{i} \delta\left(r-r_{i}\right)
$$




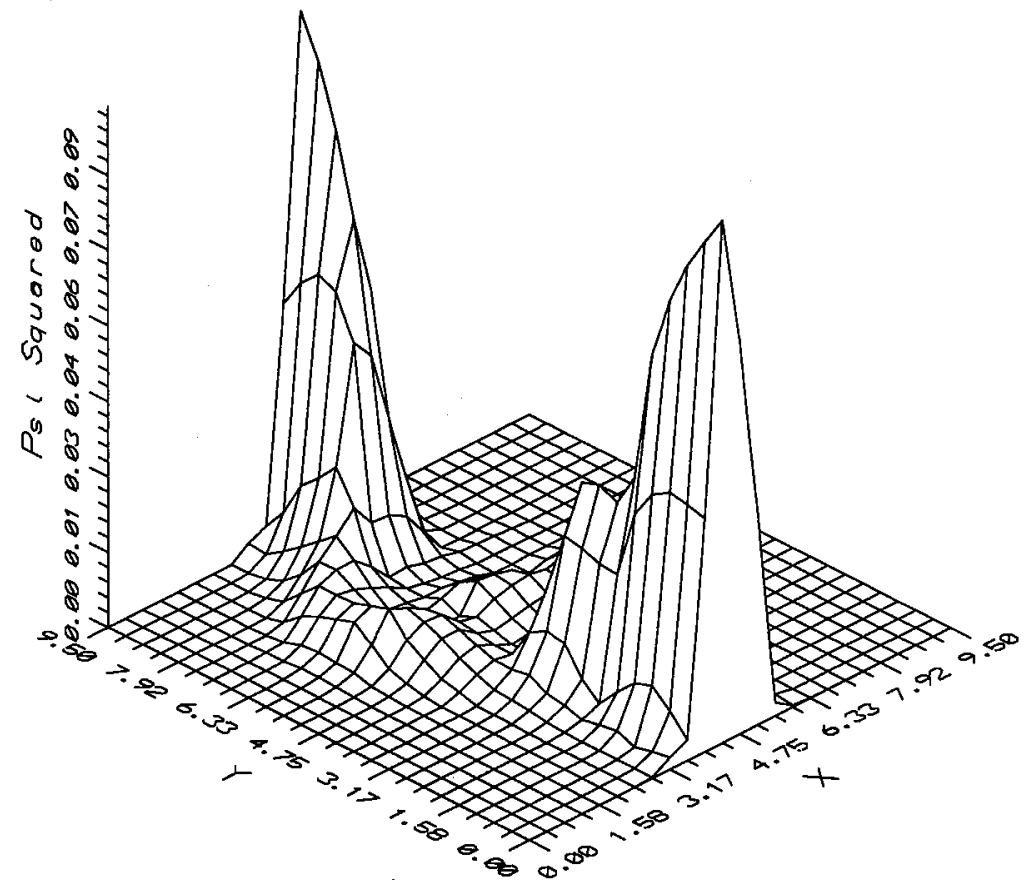

Fig. 1. The modulus square of scattering wave function. Magnetic length $l$ is used as a length unit $\left(l^{2}=h / 2 \pi e B\right)$.

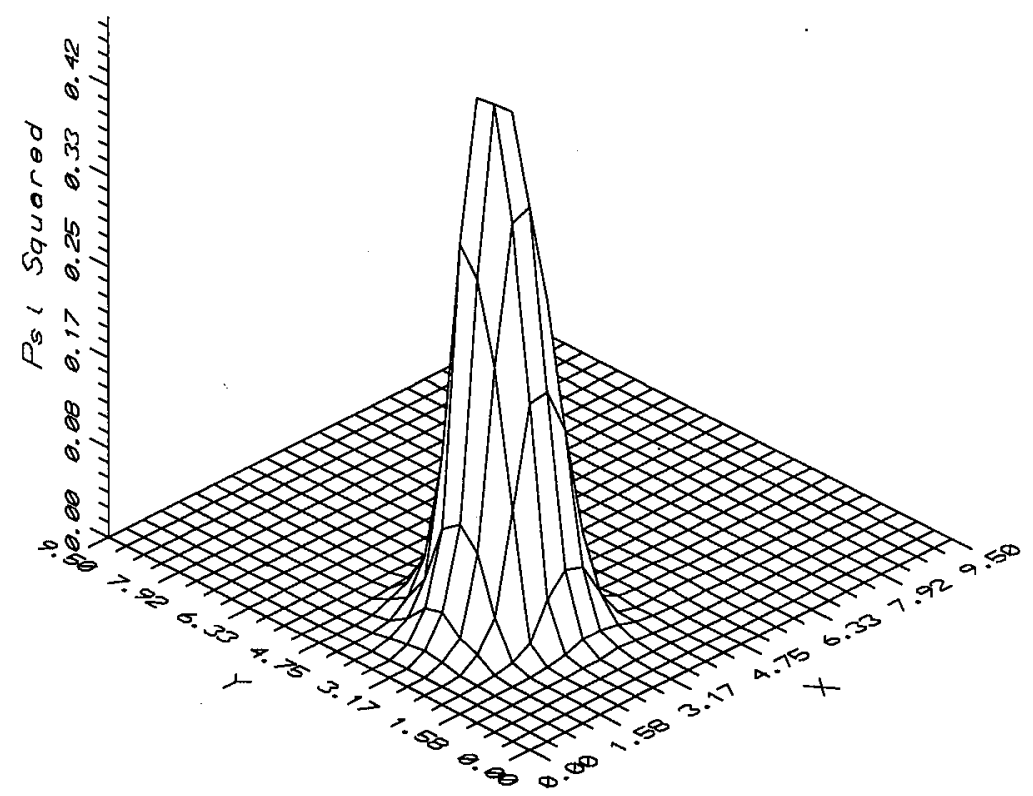

Fig. 2. The modulus square of the bound state wave function at energy $\varepsilon / \hbar \omega_{c}=0.06$. 
In Fig. 1 we plot the modulus square of the wave function obtained for $N=10$ short ranged scatterers placed randomly in the central region of the sample. It can be easily seen that asymptotically the wave is up to (possibly) phase factor equal to unperturbed one and moreover the asymptotics is reached few magnetic length after the last scatterer. This behaviour is in agreement with previous predictions [4].

Figure 2 shows example of the low energy $\varepsilon=0.06 \hbar \omega_{c}$ bound state, obtained for the same distribution of scatterers.

\section{References}

[1] K. von Klitzing, G. Dorda, B. Pepper, Phys. Rev. Lett. 45, 449 (1980).

[2] see e.g. The Quantum Hall Effect, Eds. R.E. Prange, S.M. Girvin, Springer Verlag, Berlin 1987.

[3] W. Brenig, Z. Phys. B 50, 305 (1983).

[4] W. Brenig, K.I. Wysokiński, Z. Phys. B 63, 149 (1986).

[5] T. Ando, Surf. Sci. 113, 182 (1982); H. Aoki, J. Phys. C 16, 1893 (1983); M.Ya. Azbel, O. Entin-Wohlman, Phys. Rev. B 32, 562 (1985). 